\title{
A topological spin glass in diluted spin ice
}

\author{
Arnab Sen ${ }^{1,2}$ and R. Moessner ${ }^{2}$ \\ ${ }^{1}$ Department of Theoretical Physics, Indian Association for the Cultivation of Science, Kolkata-700032, India \\ ${ }^{2}$ Max-Planck-Institut für Physik komplexer Systeme, 01187 Dresden, Germany
}

(Dated: July 9, 2018)

\begin{abstract}
It is a salient experimental fact that a large fraction of candidate spin liquid materials freeze as the temperature is lowered. The question naturally arises whether such freezing is intrinsic to the spin liquid ("disorder-free glassiness") or extrinsic, in the sense that a topological phase simply coexists with standard freezing of impurities. Here, we demonstrate a surprising third alternative, namely that freezing and topological liquidity are inseparably linked. The topological phase reacts to the introduction of disorder by generating degrees of freedom of a new type (along with interactions between them), which in turn undergo a freezing transition while the topological phase supporting them remains intact.
\end{abstract}

Topology, liquidity and glassiness: The search for topological states of matter in magnetism over the last two decades has produced a good number of candidate classical and quantum spin liquids $\$^{1}$ which show no conventional ordering down to temperatures much lower than the energy scale $\Theta_{W}$ of their interactions. In experiment, such behaviour often goes along with glassy behavior, be it for Ising 2 or Heisenberg 3 spins; in dimensions $d=2^{31617}$ or $d=3^{214}$; and for different disorder types, e.g. distortions ${ }^{8}$ or dilution ${ }^{5}$. In this way, the study of glassy physics has become one of the staples of the field. A comprehensive discussion is provided in Ref. 9.

A systematic understanding of the rich experimental findings has been slow to emerge. Even the minimal ingredients to obtain freezing remain unclear. A seductive idea was the prospect of disorder-free glassiness, where a rugged energy landscape was posited to exist even in the absence of quenched disorder, thus accounting for the slow dynamics910. A more pedestrian alternative is the realist view that any system exhibits some level of quenched disorder, and hence a tendency towards glassy behaviour, which becomes frequently visible in the case of spin liquids as there, it is not preempted by other instabilities. Indeed, in the case of bond disorder, $\frac{11}{11}$ the existence of a conventional glass transition in the pyrochlores was shown to occur at a critical temperature set simply by the amplitude of the bond disorder $\frac{12}{}$

Our work presents a third way towards glassiness in topological spin states: the interplay of disorder with the topological phase produces emergent degrees of freedom along with interactions between them; it is these new degrees of freedom which in turn undergo a freezing transition. This combination of "extrinsic" disorder teaming up with "intrinsic" properties of the topological state presents an attractive conceptual angle on the ubiquity of spin freezing in those systems; we call the resulting state 'topological spin glass', as spin freezing emerges from a substrate of a topological spin liquid, in our example the topological Coulomb phase of spin ice.

Phase diagram of diluted spin ice: Our central result is the phase diagram, Fig. 11, which shows the onset of the topological Coulomb regime at a temperature set by the cost of a topological defect (magnetic monopole)

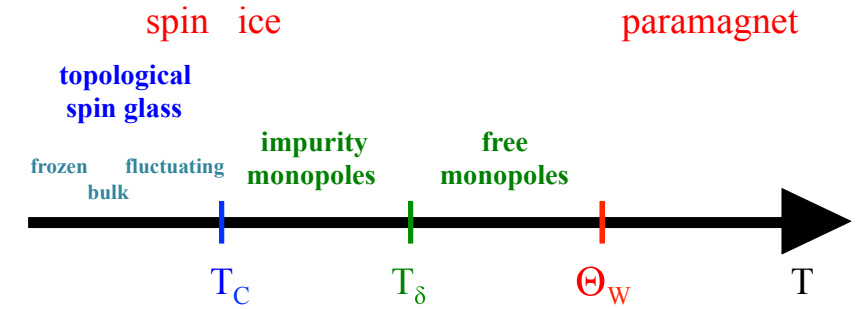

FIG. 1: Phase diagram of spin ice with a small density $x$ of spins removed. The glass transition takes place at $T_{c}$, while the crossovers from the high-temperature paramagnet to the topological Coulomb phase, and to impurity dominated monopole excitations take place at $\Theta_{W}$ and $T_{\delta}$, respectively.

$\Theta_{W} \sim \Delta$. At a lower temperature, $\mathrm{T}_{c}$-proportional to the dilution (density of missing spins) $x$-there is a spin glass transition. Below $\mathrm{T}_{c}$, the correlations characteristic of the topological Coulomb phase persist alongside a small frozen moment.

Remarkably, our theory is very simple when cast in terms of missing spins, which we call ghost spins. This occurs much in the same way that an almost filled band of electrons is most simply described in terms of dilute, positively charged holes, i.e. missing electrons.

The dumbbell model and ghost spins: In dipolar spin ice, the degrees of freedom are Ising spins on the pyrochlore lattice (Fig. 2 whose local easy axis directions, $\hat{e}_{i}$, are defined by the line joining the centres of the pair of tetrahedra which share them; the simplest appropriate interaction Hamiltonian of Ising spins with moments $\vec{\mu}_{i, j}$ of size $\mu$, separated by $r_{i j}$, contains short-range exchange interactions in addition to the usual magnetic dipolar term, $D \mathcal{D}_{i j}$, of strength $D$, with

$$
\mathcal{D}_{i j}=\frac{1}{\mu^{2}}\left(\frac{a}{r_{i j}}\right)^{3}\left(\vec{\mu}_{i} \cdot \vec{\mu}_{j}-3\left(\vec{\mu}_{i} \cdot \hat{r}_{i j}\right)\left(\vec{\mu}_{j} \cdot \hat{r}_{i j}\right)\right)
$$

with $a$ the nearest neighbour distance on the pyrochlore lattice, and $a_{d}=a \sqrt{3 / 2}$ the separation between the centres of the tetrahedra, which define a diamond lattice.

To derive our central results, we appeal to the equiva- 


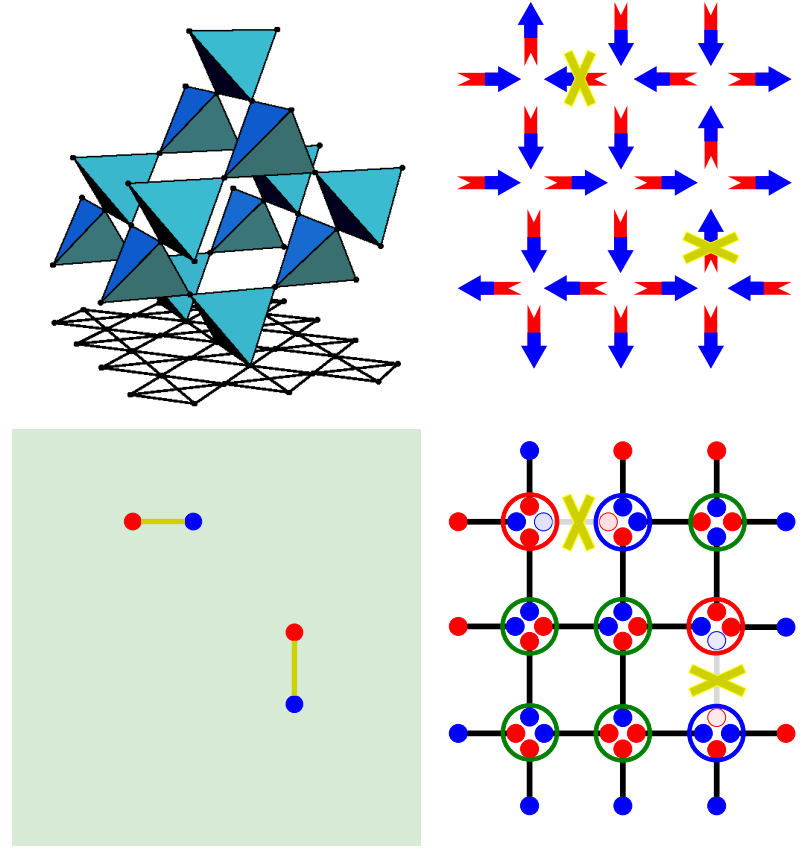

FIG. 2: Complexity reduction via genesis of ghost spins. The cartoons are for a projection of spin ice onto the twodimensional plane (top left panel) for clarity; the diamond lattice defined by the centres of the tetrahedra thus turns into a square lattice. At low temperature $T \ll \delta, \Delta$, spin ice with a small density $x$ of missing spins (crossed out in top right) is equivalent to a small density of ghost spins (bottom left). This is straightforwardly established by writing each spin as a dumbbell of equal and opposite magnetic charges. At vertices either end of a missing spin, the net charge is nonzero (red and blue circles), so that they form the ends of the dipole of the ghost spin. At all other vertices, the net charge vanishes (green circles) and can thus be omitted, so that this pristine bulk of spin ice only provides an effective medium (shaded green) carrying an interaction between the ghost spins, Eq. 3 and Fig. 3 .

lent dumbbell model introduced in the prediction of the existence of magnetic monopoles ${ }^{[13}$ Here, each spin is represented by two equal and opposite magnetic charges $\mathcal{Q}=\mu / a_{d}$. The only details of the model we require ${ }^{13}$ is that the pairwise spin interactions can be rewritten as a pairwise interaction of the total charges of each tetrahedron, $\mathcal{Q}_{\text {tet }}$. This includes a contact interaction $\Delta\left(a_{d} / 2 \mu\right)^{2} \sum_{t e t} \mathcal{Q}_{\text {tet }}^{2}$ to reproduce the nearest neighbour interaction correctly, in addition to standard (magnetic) Coulomb interactions between any other pair of charges. From these and the nearest neighbour exchange $J$, we can construct two energy scales; $D=\mu_{0} \mu^{2} /\left(4 \pi a^{3}\right)$, the coupling constant of the dipolar interaction (with vacuum permeability $\left.\mu_{0}\right)$ and $\Delta=\frac{2 J}{3}+\frac{8}{3}\left(1+\sqrt{\frac{2}{3}}\right) D$, the energy cost of a $\left|\mathcal{Q}_{\text {tet }}\right|=2 \mathcal{Q} \neq 0$ defect.

Crucially, any of the exponentially many configurations satisfying the ice rule - that two spins point into each tetrahedron and two out ${ }^{14}$ - is an exact ground state

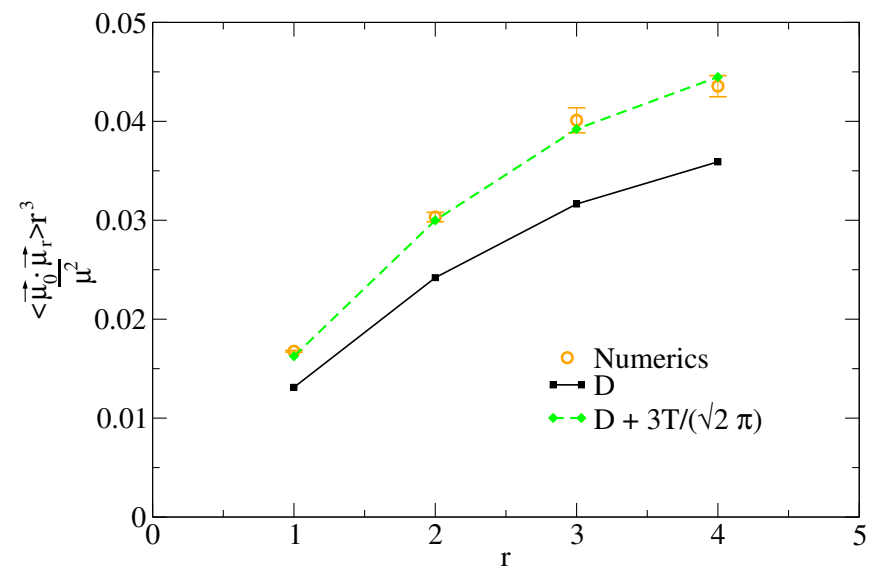

FIG. 3: Correlations between a pair of ghost spins $\vec{\mu}_{0}$ and $\vec{\mu}_{r}$ in an otherwise fully populated sample of spin ice (of linear dimension $L=12$, with $D=1.41 \mathrm{~K}$ and $T=0.5 \mathrm{~K}$ ), separated by $r$ units in the [101] direction. This is compared to two isolated magnetic dipoles in an otherwise empty unit cell; agreement with numerics is only achieved upon including the entropic interactions mediated by the spin ice bulk, for which the dashed line denotes the asymptotic form, Eq. 3

of this model. This amounts to each diamond lattice site being charge neutral: in any ground state, the charge density vanishes locally $y^{13}$.

This is the basis for what follows. Removing a spin by chemical substitution of a magnetic by a non-magnetic ion, leaves behind two adjacent tetrahedra with equal and opposite charges $\pm \mathcal{Q}$, a dipole $-\vec{\mu}$ - the ghost spin with moment opposite to that of the removed spin. Just like the charge of a hole in a semiconductor being the opposite of that of the missing electron, here it is the spin's magnetic moment which has changed sign.

The effective energetics follows simply by keeping track of the interactions between those charges - the pairwise interaction between separated ghost spins, $\tilde{\mathcal{H}}_{i j}$ has the standard dipolar form (again in complete analogy to the Coulomb repulsion between holes in a semiconductor):

$$
\tilde{\mathcal{H}}_{i j}=\tilde{D} \mathcal{D}_{i j}
$$

Note the tremendous complexity reduction - a dense, weakly diluted system of dipolar spins is described in terms of a low density of ghost spins! However, the intricate nature of the spin ice phase has not vanished entirely. The following are its most striking manifestations.

Firstly, the fractionalised excitations of the topological spin ice phase can be nucleated at the missing spin. In detail, a pair of ghost spins can be turned into a pair of impurity magnetic monopoles by flipping a string of spins arranged head-to-tail running between them. For well-separated monopoles, the resulting configuration is higher in energy by $\delta=4 \sqrt{2} D /(3 \sqrt{3})$ per monopole.

Secondly, the ghost spins do not disrupt the correlations in spin ice, as they have zero net magnetic charge, so that in particular the pinch-points ${ }^{15}$ found in neu- 
tron scattering, which reflect the emergent gauge field defining the Coulomb phase, remain intact.

Thirdly, the effective dipolar coupling constant between the ghost spins $\tilde{D}$, Eq. 2 , has a contribution coming from the fluctuations of the spins in the bulk on top of the simple magnetostatic coupling constant $D$ :

$$
\tilde{D}=D+\frac{3 T}{\sqrt{2} \pi}
$$

This happens because the number of spin ice states compatible with a given configuration of ghost spins depends on their relative orientation; this yields an entropic contribution, $J^{\text {ent }}$, to the spin interaction ${ }^{15}$ - for details, see the Suppl. Mat., where we derive the expression

$$
J_{i j}^{\mathrm{ent}}=-T\left\langle\sigma_{i} \sigma_{j}\right\rangle_{\mathrm{nn}},
$$

where $\left\langle\sigma_{i} \sigma_{j}\right\rangle_{\mathrm{nn}}$ stands for the correlations between Ising spins on sites $i$ and $j$ in spin ice with the dipolar interactions switched off entirely; this gives Eq. 3 for $r_{i j} \gg a_{d}$ but is accurate also for small $r_{i j}$ (Fig. 3).

In other words, thanks to the Coulomb phase, the missing dipolar spins know about the correlations they would have if they were neither missing nor dipolar!

Freezing of the ghost spins: It is notoriously hard to simulate spin freezing transitions $\frac{18}{20}$, all the more so in this case where a small number of ghost spins requires simulation of a much larger number of bulk spins. However, thanks to the abovementioned complexity reduction, this effective problem, Eq. 2, can be analysed, still with a considerable amount of numerical effort.

We have demonstrated numerically that there is spin freezing for the random dipolar model, Fig. 4. We find a critical temperature $\mathrm{T}_{x} \propto x$, as one would expect for a dipolar system with typical distance between spins $r \sim x^{-1 / 3}$, and hence dipolar interaction energy scale $\sim r^{-3} \sim x$. We find numerically that $\mathrm{T}_{x} \simeq$ $0.95 D x$, which implies an entropically renormalised value of $T_{c}(x)=T_{x} /\left(1-\frac{3 T_{x}}{\sqrt{2} \pi D}\right)$ for the freezing transition into the topological spin glass.

This complements known cases of freezing for random dipoles, namely dense dipoles on a cubic lattice with random orientations, $; 12$ or dilute but collinear dipoles on a cubic lattice. ${ }^{22}$ In our case, the dipoles are dilute and their orientations are neither random nor collinear, being picked from the local easy axes of the occupied sites, respecting the cubic symmetry of the pyrochlore lattice.

Energy scales and the role of perturbations: Let us now examine the phase diagram Fig. 1 in more detail. At high temperature, $\mathrm{T} \gg \Delta$, we have an ordinary disordered paramagnet. Below $\Theta_{W} \sim \Delta$, the ice rules are enforced, yielding the topological Coulomb regime with experimentally sparse monopoles as elementary excitations, $\rho \sim e^{-\Delta / T ! 13}$ However, the impurity magnetic monopoles mentioned above have a cheaper energy $\operatorname{cost} \delta<\Delta$ than a monopole in the bulk as no energy needs to be paid for violating the ice rule in the first place - this is taken care of by the quenched chemical dilution. The corresponding

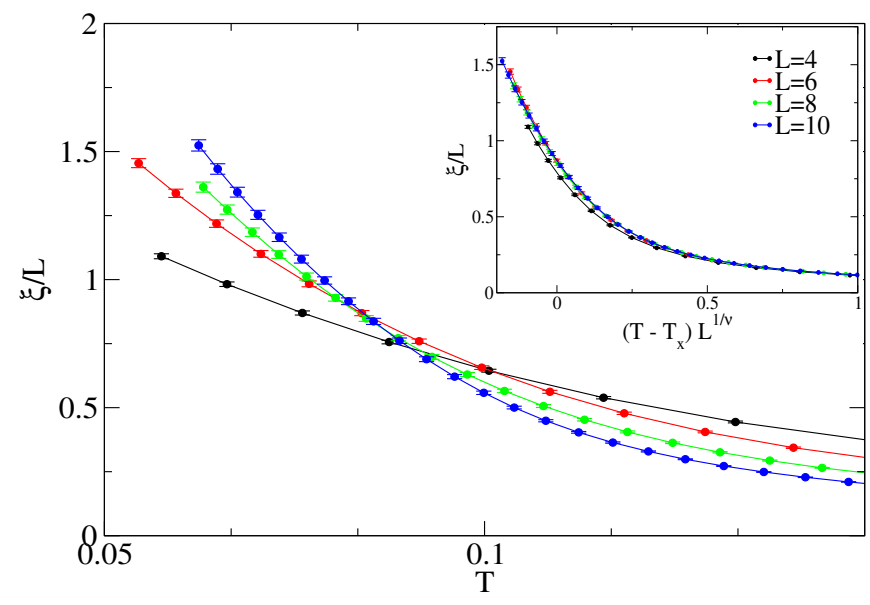

FIG. 4: The freezing transition. Number of spins equals $16 L^{3} x$ in a system with $L^{3}$ unit cells, with $x=0.0625$. Dipolar coupling of $D=1.41 \mathrm{~K}$ (as in $\mathrm{Ho}_{2} \mathrm{Ti}_{2} \mathrm{O}_{7}$ and $\left.\mathrm{Dy}_{2} \mathrm{Ti}_{2} \mathrm{O}_{7}\right)^{\sqrt{14}}$ is used in simulations: the crossing point in $\xi / L$ (see Suppl. Mat.) indicates a continuous glass transition, further evidence for which is the data collapse (inset) for different system sizes, for $\nu=1.08(3)$ and critical temperature $T_{x}=0.084(2)$.

density is $\rho_{x} \sim x e^{-\delta / T}$, which dominates over $\rho$ at lower temperatures below $T_{\delta}=(\delta-\Delta) / \ln x$. Further, at $T_{c}$, the glass transition occurs, at which (presumably 23124 ) Ising symmetry is broken. The bulk spins in the Coulomb phase continue to fluctuate, however.

This is the above-mentioned coexistence of the topological phase and the spin glass phase for which it provides the substrate-the topological spin glass.

The frozen moment - the 'order parameter' $q_{E A}=$ $\frac{1}{N} \sum_{i=1}^{N}\left\langle S_{i}\right\rangle^{2}$ of the spin glass - appears at $T_{c}$ and grows as the temperature is lowered, as do static local fields set up by the frozen moments $B_{f} \sim\left(\mu_{0} \mu x \sqrt{q_{E A}}\right) /\left(4 \pi a^{3}\right)^{25}$. The resultant Zeeman energy $\sim D \sqrt{q_{E A}} x$ will try to pin the bulk spins to point along the direction of the local fields, against the entropy of fluctuations between different spin ice configurations, ${ }^{26}$ estimated by Pauling to be $S_{p} \approx \frac{1}{2} \ln \frac{3}{2}$ per spin. The glass transition at $T_{c}$ being continuous, for $T \lesssim T_{c}$ the frozen moment and concomitant Zeeman energies will be very small, and the bulk spins will continue to fluctuate essentially like in pure spin ice. As $T$ is lowered further, the entropic contribution of the bulk fluctuations to the free energy vanishes approximately $\propto T$, while $q_{E A}$ grows, so that the system eventually freezes into one spin ice configuration. Since the static fields are too weak to break the ice rules, the frozen state still exhibits the correlations of the Coulomb phase when averaged over the entire sample $e^{1427}$.

Comments on experiment and outlook: Generally speaking, the low-temperature physics of frustrated systems is non-universal, and the topological spin glass may be preempted by perturbations to the dumbbell Hamiltonian if they are large enough. Indeed, the question what happens in diluted spin ice compounds ${ }^{28}$ was recently addressed in detailed numerical simulations, ${ }^{29}$ which em- 
phasized the need to consider the complete Hamiltonian to obtain a fit of theory to experiment. The question of whether freezing generically occurs was not settled there.

The central point of our work is that there exists a microscopic Hamiltonian for which the existence of the topological spin glass can be predicted with a high degree of confidence. Features such as the simultaneous appearance of topologically spin liquidity and glassiness appear naturally, along with the presence of a small frozen moment alongside a sizeable fluctuating component. The challenge for establishing its existence in experiment is thence to find a compound avoiding other instabilities both of the non-dynamic and dynamic nature, such as a non-cooperative slowing down. 30 This seems a very realistic prospect given the wide range of spin ices available nowadays, with the combination alone of $\mathrm{A}_{2} \mathrm{~B}_{2} \mathrm{O}_{7}$, $(\mathrm{A}=\mathrm{Dy}, \mathrm{Ho}, \mathrm{Tb}, \mathrm{Yb}, \ldots, \mathrm{B}=\mathrm{Ti}, \mathrm{Ge}, \mathrm{Sn}, \ldots)$ providing numerous examples differing in many fundamental parameters such as the relative size of exchange and dipolar interactions, as well as many single-ion properties.

More broadly, we would like to emphasize the genericity of the ingredients involved in our study. We used the "vacuum" of a Coulomb phase and its local charge neutrality, and the fact that defects therein will have an interaction determined by the emergent gauge theory describing the low energy physics of the topological phase. The detailed resulting collective behaviour will be as varied as the richness of the latter ingredients; therefore, it is clear that an outcome in which randomly located emergent degrees of freedom interact via highly frustrated interactions is a generic one, and so is the expectation of a topological spin glass.

Acknowledgements: We are very grateful to Michel Gingras, Shivaji Sondhi and Claudio Castelnovo for useful discussions, to the latter two for collaboration on related work, and to the last for detailed comments on the manuscript.
1 P. A. Lee, An end to the drought of spin liquids, Science 321 1306-1307 (2008).

2 J. Snyder, J. S. Slusky, R. J. Cava, and P. Schiffer, How 'spin ice' freezes, Nature (London) 413, 48 (2001).

3 A. P. Ramirez, G. P. Espinosa, and A. S. Cooper, Strong frustration and dilution-enhanced order in a quasi-2D spin glass, Phys. Rev. Lett. 64, 2070 (1990).

4 P. Schiffer, A. P. Ramirez, D. A. Huse, P. L. Gammel, U. Yaron, D. J. Bishop, and A. J. Valentino, Frustration induced spin freezing in a site-ordered magnet: Gadolinium Gallium Garnet, Phys. Rev. Lett. 74, 2379 (1995).

5 A. D. LaForge, S. H. Pulido, R. J. Cava, B. C. Chan, and A. P. Ramirez, Quasispin glass in a geometrically frustrated magnet, Phys. Rev. Lett. 110, 017203 (2013).

6 A. S. Wills, A. Harrison, C. Ritter, and R. I. Smith, Magnetic properties of pure and diamagnetically doped jarosites: Model kagomé antiferromagnets with variable coverage of the magnetic lattice, Phys. Rev. B 61, 6158 (2000).

7 F. Bert, D. Bono, P. Mendels, F. Ladieu, F. Duc, J.-C. Trombe, and P. Millet, Ground state of the kagomé-like $S=1 / 2$ antiferromagnet Volborthite $\mathrm{Cu}_{3} \mathrm{~V}_{2} \mathrm{O}_{7}(\mathrm{OH})_{2} .2 \mathrm{H}_{2} \mathrm{O}$, Phys. Rev. Lett. 95, 087203 (2005).

8 H. J. Silverstein, K. Fritsch, F. Flicker, A.M. Hallas, J.S. Gardner, Y. Qiu, G. Ehlers, A.T. Savici, Z. Yamani, K.A. Ross, B.D. Gaulin, M.J.P. Gingras, J.A.M. Paddison, K. Foyevtsova, R. Valenti, F. Hawthorne, C.R. Wiebe, H.D. Zhou, Liquidlike correlations in single-crystalline $\mathrm{Y}_{2} \mathrm{Mo}_{2} \mathrm{O}_{7}$ : An unconventional spin glass, Phys. Rev. B 89, 054433 (2014).

9 O. Cepas, B. Canals, Heterogeneous freezing in a geometrically frustrated spin model without disorder: spontaneous generation of two time-scales, Phys. Rev. B 86, 024434 (2012).

10 P. Chandra, P. Coleman, and I. Ritchey, The anisotropic kagome antiferromagnet: a topological spin glass?, J. Phys. I France 3, 591 (1993).

11 L. Bellier-Castella, M. J. P. Gingras, P. C. W. Holdsworth and R. Moessner, Frustrated order by disorder: The py- rochlore anti-ferromagnet with bond disorder, Can. J. Phys. 79, 1365 (2001).

12 T. E. Saunders and J. T. Chalker, Spin freezing in geometrically frustrated antiferromagnets with weak disorder, Phys. Rev. Lett. 98, 157201 (2007).

13 C. Castelnovo, R. Moessner and S. L. Sondhi, Magnetic monopoles in spin ice, Nature 451, 42 (2008).

14 S. T. Bramwell and M. J. P. Gingras, Spin ice state in frustrated magnetic pyrochlore materials, Science 294, 1495 (2001).

15 S. Isakov, R. Moessner and S. L. Sondhi, Dipolar spin correlations in classical pyrochlore magnets, Phys. Rev. Lett. 93, 167204 (2004).

16 A. Sen, R. Moessner, and S. L. Sondhi, Coulomb phase diagnostics as a function of temperature, interaction range and disorder, Phys. Rev. Lett. 110, 107202 (2013).

17 T. Fennell, P. P. Deen, A. R. Wildes, K. Schmalzl, D. Prabhakaran, A. T. Boothroyd, R. J. Aldus, D. F. McMorrow, S. T. Bramwell, Magnetic Coulomb Phase in the Spin Ice $\mathrm{Ho}_{2} \mathrm{Ti}_{2} \mathrm{O}_{7}$, Science 326, 415 (2009).

18 K. Hukushima and K. Nemoto, Exchange Monte Carlo method and application to spin glass simulations, J. Phys. Soc. Jpn. 65, 1604 (1996).

19 M. Hasenbusch, A. Pelissetto, and E. Vicari, Critical behaviour of three-dimensional Ising spin glass models, Phys. Rev. B 78, 214205 (2008).

20 L. A. Fernandez, V.M.-Mayor, S.P.-Gaviro, A. Tarancon, and A. P. Young, Phase transition in the three dimensional Heisenberg spin glass: Finite-size scaling analysis, Phys. Rev. B 80, 024422 (2009).

21 J. F. Fernández, Monte Carlo study of the equilibrium spinglass transition of magnetic dipoles with random anisotropy axes, Phys. Rev. B 78, 064404 (2008).

${ }^{22}$ K. Tam and M. J. P. Gingras, Spin-glass transition at non-zero temperature in a disordered dipolar Ising system: The case of $\mathrm{LiHo}_{x} \mathrm{Y}_{1-x} \mathrm{~F}_{4}$, Phys. Rev. Lett. 103, 087202 (2009).

23 J. R. L. de Almeida and D. J. Thouless, Stability of the Sherrington-Kirkpatrick solution of a spin glass model, J. 
Phys. A: Math. Gen. 11, 983 (1978).

24 D. Larson, H. G. Katzgraber, M. A. Moore, A. P. Young, Spin glasses in a field: Three and four dimensions as seen from one space dimension, Phys. Rev. B 87, 024414 (2013).

25 N. Rivier and K. Adkins, in Amorphous Magnetism, edited by H. O. Hooper and A. M. de Graaf (Plenum, New York, 1973), p. 215.

26 A. P. Ramirez, A. Hayashi, R. J. Cava, R. Siddharthan and B. S. Shastry, Zero-point entropy in 'spin ice', Nature 399, 333-335 (1999).

27 C. Castelnovo, R. Moessner and S. L. Sondhi, Spin Ice, Fractionalization, and Topological Order, Annual Rev. of Cond. Matt. Phys. 3, 35 (2012).

${ }^{28}$ X. Ke, R. S. Freitas, B. G. Ueland, G. C. Lau, M. L. Dahlberg, R. J. Cava, R. Moessner, and P. Schiffer, Nonmonotonic Zero-Point Entropy in Diluted Spin Ice, Phys. Rev. Lett. 99, 137203 (2007).

29 T. Lin, X. Ke, M. Thesberg, P. Schiffer, R. G. Melko, M. J. P. Gingras, Non-monotonic residual entropy in diluted spin ice: a comparison between Monte Carlo simulations of diluted dipolar spin ice models and experimental results, arXiv:1303.7240 (2013).

${ }^{30}$ I. A. Ryzhkin, Magnetic relaxation in rare-earth oxide pyrochlores, JETP 101, 481 (2005).

31 L. D. C. Jaubert and P. C. W. Holdsworth, Signature of magnetic monopole and Dirac string dynamics in spin ice, Nat. Phys. 5, 258 (2009).

32 see e.g. M. J. P. Gingras, P. A. McClarty, preprint arXiv:1311.181\%.

\section{Appendix A: Derivation of the effective Hamiltonian}

We first establish Eq. 3. that the ghost dipoles experience an enhanced interaction compared to free dipolar spins, mediated by the spin ice bulk. This form is valid at low temperature, where monopoles are exponentially sparse so that they can be neglected to an excellent approximation. Our demonstration consists of showing that the dipolar and entropic part of the interactions simply add; and that the latter is reflected in the correlations of spins in 'nearest-neighbour' spin ice, i.e. in the absence of magnetic dipolar interactions and disorder.

For this case, we denote the Ising spins with variables $\sigma_{i}$. It is a non-trivial but known fact that the correlations induced by the entropic interactions are dipolar, 15 with

$\left\langle\sigma_{i} \sigma_{j}\right\rangle_{T=0}=-\frac{3}{\sqrt{2} \pi}\left(\frac{a}{r_{i j}}\right)^{3}\left(\hat{e}_{i} \cdot \hat{e}_{j}-3\left(\hat{e}_{i} \cdot \hat{r}_{i j}\right)\left(\hat{e}_{j} \cdot \hat{r}_{i j}\right)\right)$,

where the numerical prefactor $3 / \sqrt{2} \pi$ is computed approximately but accurately from a large- $n$ ansatz.

This result is reproduced by a simple pairwise effective free energy, which keeps track of the relative number of spin ice states compatible with the four possible configuration of the spin pair:

$$
\mathcal{F}_{\sigma_{i} \sigma_{j}}=J_{i, j}^{\mathrm{ent}} T \sigma_{i} \sigma_{j} \Rightarrow-T\left\langle\sigma_{i} \sigma_{j}\right\rangle_{T=0}=J_{i, j}^{\mathrm{ent}},
$$

which follows upon linearising the Boltzmann factor $\exp (-\mathcal{F} / T)$, which is appropriate as these correlations are small. These equations together establish an entropic coupling constant $3 T / \sqrt{2} \pi$, which as we show next is the difference between $\tilde{D}$ and $D$.

Let us now consider the full dipolar interaction problem and reinsert a dipole each at the location of the two ghost spins as to obtain a state obeying the ice rules. In the absence of monopoles at low temperature, this is always possible, crucially, in a unique way. This means that the counting of the number of configurations compatible with a given state of the ghost spins does not depend on their presence or absence, so that the (unnormalised) Boltzmann factor for a given ghost spin configuration is simply the multiplicity - given by the entropic free energy, Eq. A2 - multiplied by the energetic Boltzmann factor due to the dipolar interaction. Together with Eq. 2, we thus obtain the full interaction between the ghost spins as

$$
\left(D+\frac{3 T}{\sqrt{2} \pi}\right)\left(\frac{a}{\mu r_{i j}}\right)^{3}\left(\hat{\mu}_{i} \cdot \hat{\mu}_{j}-3\left(\hat{\mu}_{i} \cdot \hat{r}_{i j}\right)\left(\hat{\mu}_{j} \cdot \hat{r}_{i j}\right)\right),
$$

(using $\hat{\mu}_{i} \| \hat{e}_{i}$ and $\vec{\mu}_{i}=\mu \hat{\mu}_{i}$ ); this gives Eq. 3 .

\section{Appendix B: Freezing transition at small dilution}

We use Monte-Carlo simulations to study the freezing transition in very dilute spin ice, obtained after mapping the weakly diluted system of dipolar spins to a low density of ghost spins, and show that there is a continuous transition to a spin glass phase. A single-spin flip Metropolis algorithm in combination with parallel tempering in temperature ${ }^{18}$ is used to ensure proper equilibration in the glassy phase. The data is averaged over several disorder realizations (typically 1000 or more), each of which is produced by placing spins on a fraction $x$ of sites that are randomly selected from a total of $16 L^{3}$ sites in a system of linear size $L$ (with 16 sites in the conventional cubic unit cell for the pyrochlore lattice). The long-ranged nature of the dipolar interactions is treated using the Ewald summation technique (e.g. see Z.Wang and C. Holm, J. Chem. Phys. 115, 6351 (2001)).

The spin glass order parameter, $q_{E A}^{\alpha \beta}(\mathbf{k})$, at wavevector $\mathbf{k}$ is defined as

$$
q_{E A}^{\alpha \beta}(\mathbf{k})=\frac{1}{N} \sum_{i} \mu_{i}^{\alpha(1)} \mu_{i}^{\beta(2)} \exp \left(i \mathbf{k} \cdot \mathbf{r}_{i}\right)
$$

where $N=16 L^{3} x ; \alpha, \beta=x, y, z$ are the spin components (where the ghost spins point along the local easy axes) and (1) and (2) denote two identical disorder realizations of the system with the same set of interactions. From this, we calculate the spin glass susceptibility, $\chi_{S G}(\mathbf{k})$, defined as

$$
\chi_{S G}(\mathbf{k})=N \sum_{\alpha, \beta}\left[\left\langle\left|q_{E A}^{\alpha \beta}(\mathbf{k})\right|^{2}\right\rangle\right]
$$

where $\langle\cdots\rangle$ and $[\cdots]$ denote thermal and disorder averages, respectively. 


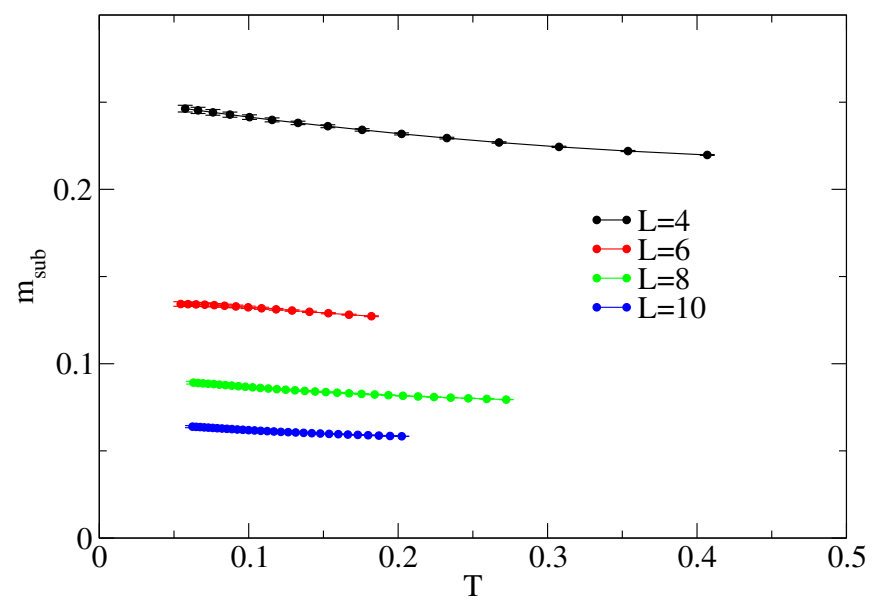

FIG. 5: The behaviour of sublattice magnetization $m_{\text {sub }}$ for $x=0.0625$ at coupling $D=1.41 \mathrm{~K}$. The data shows the absence of any long-range ordering of $m_{s u b}$ on lowering the temperature below the glass transition $T_{x}$.

A spin glass correlation length $\xi$ can then be defined by using the following relation:

$$
\xi=\frac{1}{2 \sin \left(\frac{\left|\mathbf{k}_{\min }\right|}{2}\right)}\left(\frac{\chi(0)}{\chi\left(\mathbf{k}_{\min }\right)}-1\right)^{\frac{1}{2}}
$$

where $\mathbf{k}_{\min }=\frac{2 \pi}{L}(1,0,0)$. If the freezing transition is continuous, then $\xi$ is expected to satisfy a scaling relation of the form $\xi / L=\mathcal{F}\left(\left(T-T_{x}\right) L^{1 / \nu}\right)$ where $\mathcal{F}$ is a universal scaling function and $T_{x}$ is the critical temperature. From this, it follows that $\xi / L$ for different sizes $L$ (for sufficiently large $L$ ) should cross at $T_{x}$. The behaviour of $\xi / L$ from the numerics for small $x$ indeed confirms to this expectation (see Fig 4 ) and provides numerical evidence for a continuous spin glass transition in a dilute dipolar spin ice.

We have also checked the behaviour of other quantities to rule out any obvious long-ranged ordered states. For example, we evaluate the sublattice magnetization per site, $m_{\text {sub }}$, defined as

$$
m_{s u b}=\left[\left\langle\left|\frac{1}{N_{a}} \sum_{i} S_{i, a}\right|\right\rangle\right]
$$

where the sum runs only over sites that belong to the same sublattice $a$ (the pyrochlore lattice is a FCC lattice with a four-point basis), $N_{a}$ counts the sites in sublattice $a$ for a given disorder realization and $S_{i}=\left(\vec{\mu}_{i} \cdot \hat{e}_{i}\right) / \mu=$ \pm 1 . In Fig. 5, we see that $m_{s u b}$ scales as $L^{-3 / 2}$ with increasing $L$, indicating the absence of any ordering transition to a state with non-zero sublattice magnetization. 